\begin{tabular}{|c|l|}
\hline Title & Equality of Higher-Order Diffusion Coefficients between Component and Composite Electron Swarms in Gas \\
\hline Author(s) & Sugawara, Hirotake; Sakai, Y osuke \\
\hline Citation & $\begin{array}{l}\text { Japanese Journal of A pplied Physics, 45/6a), 5189-5196 } \\
\text { https://doi.org/40.1143/JAA.45.5189 }\end{array}$ \\
\hline Issue Date & 2006-06-08 \\
\hline Doc URL & http://hdl.handle.net/2115/33071 \\
\hline Rights & Copyright $\odot 2006$ The Japan Society of A pplied Physics \\
\hline Type & article (author version) \\
\hline File Information & Sugawara JJA P-2006.pdf \\
\hline
\end{tabular}

Instructions for use 


\section{Equality of Higher-Order Diffusion Coefficients between Component and Composite Electron Swarms in Gas}

Hirotake SUGAWARA and Yosuke SAKAI

Division of Electronics for Informatics, Graduate School of Information Science and Technology, Hokkaido University, North 14 West 9, Sapporo 060-0814, Japan

When an electron swarm in gas is composed of component electron swarms individually in drift equilibrium, the higher-order diffusion coefficients (HDCs) of the composite electron swarms are equal to those of the component electron swarms. We have derived this equality theoretically and have examined it by numerical simulation. The HDCs are the time derivatives of higher-order cumulants, which are quantities characterizing the shape of a spatial electron distribution. This fact seemingly indicates the dependence of the HDCs of the composite electron swarms on the arrangement of the component electron swarms. However, the equality holds irrespective of the relative positions and electron populations of the component electron swarms. We have given a consistent explanation to these facts. We have also discussed electron swarm development from dispersed or multiple electron sources that may appear in practical experiments.

KEYWORDS: electrical discharge, plasma, simulation, transport parameter, electron swarm, diffusion coefficient, drift equilibrium, cumulant, moment

\section{Introduction}

The electron diffusion coefficient in gas is an essential quantity for describing the electron transport in electrical discharges and plasmas. This quantity appears as the coefficient of the second-order gradient of the electron concentration in an electron continuity equation, with which numerical simulations are widely performed for various applications, such as processing plasmas and light sources.

A standard one-dimensional electron continuity equation is written as

$$
\frac{\partial}{\partial t} f(x, t)=\left(R-W \frac{\partial}{\partial x}+D \frac{\partial^{2}}{\partial x^{2}}\right) f(x, t),
$$

where $f(x, t)$ is the electron distribution at position $x$ and time $t, R$ is the effective ionization frequency, $W$ is the drift velocity, and $D$ is the diffusion coefficient. This equation is actually a truncated form of the following infinite series involving higher-order diffusion coefficients (HDCs):

$$
\begin{aligned}
\frac{\partial}{\partial t} f(x, t)= & \left(R-W \frac{\partial}{\partial x}+D_{2} \frac{\partial^{2}}{\partial x^{2}}-D_{3} \frac{\partial^{3}}{\partial x^{3}}\right. \\
& \left.+D_{4} \frac{\partial^{4}}{\partial x^{4}}-\cdots\right) f(x, t),
\end{aligned}
$$

where $D_{n}$ is the $n$ th-order diffusion coefficient $\left(D=D_{2}\right)$.

A study on the HDCs of electron swarms is an approach to the formalization of the electron transport phenomenon in gas. Tagashira et al ${ }^{1)}$ presented a hierarchy of the HDCs, formulating $D_{n}$ with the spatial moments of $f$. Kumar et al. ${ }^{2)}$ discussed the difference between physical models based on eqs. (1) and (2), and pointed out the relationship between $D_{3}$ and $D_{4}$ and the skewness and kurtosis of the shape of $f$. Blevin and Fletcher ${ }^{3)}$ obtained an expression of $D_{3}$ in an expansion of the electron energy distribution function in a hydrodynamic regime. Moreover, for ion diffusion in gas, Whealton and Mason ${ }^{4)}$ and Whealton ${ }^{5}$ ) evaluated the influences of $D_{3}$ and $D_{4}$, which appear as a deviation of the ion distribution from

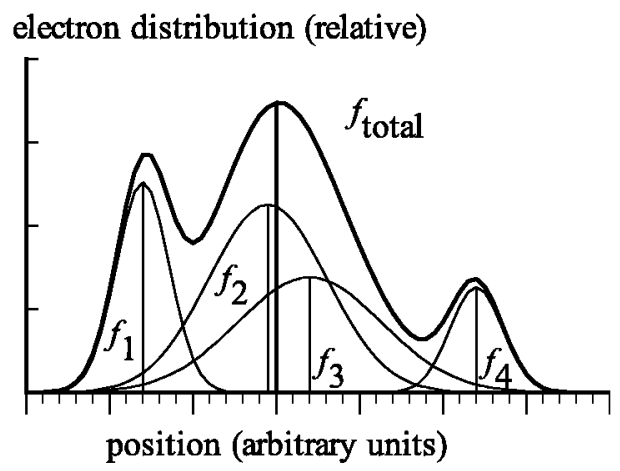

Fig. 1. Schematic of spatial electron distributions of component and composite electron swarms.

the Gaussian solution of the diffusion equation based on Fick's law.

$n ! D_{n}$ is the time derivative of the $n$ th-order cumulant $\kappa_{n}$ of $f{ }^{6}$ ) The cumulant is a statistical quantity representing the deviation of distribution shape from a Gaussian distribution. This fact indicates a mutual dependence between $D_{n}$ and the shape of $f$. Here, a question arises. Let us consider a composite electron swarm consisting of component electron swarms. In Fig. 1, $f_{k}$ $(k=1,2,3, \cdots)$ is the spatial electron distribution of the $k$ th component electron swarm. The spatial arrangement of $f_{k}$ is arbitrary and the shape of $f_{\text {total }}\left(=\sum_{k} f_{k}\right)$ of the composite electron swarm can deviate from a Gaussian as much as one intends. The cumulant $\kappa_{n}$ of $f_{\text {total }}$ is dependent on the arrangement of $f_{k}$. When the component electron swarms are individually in drift equilibrium under a common uniform electric field, i.e., when the $D_{n}$ value is common among all the component electron swarms (let the value be $D_{n, \text { eq }}$ ), is $D_{n}=\left(\mathrm{d} \kappa_{n} / \mathrm{d} t\right) / n$ ! of the composite electron swarm always equal to $D_{n, \mathrm{eq}}$ ?

Such a composite electron swarm may appear, for example, in a time-of-flight experiment using electrical shutters or UV irradiation for the release of initial electrons. When it has a temporal or spatial distribution, the 
electron swarm can be regarded as a composite electron swarm developing from multiple electron sources. The typical gate periods $1-10 \mathrm{~ns}^{7-11)}$ and shutter grid spacing $1 \mathrm{~mm}^{12)}$ can be comparable to the relaxation time and distance of electron swarms under certain conditions, although they are practically neglected by setting the observation time and distance relevantly long.

In general, the equality of transport parameters including the HDCs is expected from a thought that the electron transport parameters in drift equilibrium should be independent of initial conditions, such as the spatial arrangement of the electron sources. Therefore, the confirmation of the equality is meaningful not only for the formality of the electron swarm theory but also for the appropriate treatment of transport parameters in the practical simulations of plasma applications. In this paper, we analytically derive the equality of $D_{n}$ between the component and composite electron swarms, and examine the result via numerical calculations of $D_{n}$. We give a consistent explanation to a fact that $D_{n}=\left(\mathrm{d} \kappa_{n} / \mathrm{d} t\right) / n$ ! is independent of the arrangement of $f_{k}$, while $\kappa_{n}$ is dependent on it.

\section{Theory}

We first describe the definitions of the quantities related to the HDCs and assumptions in the present analysis. Subsequently, we derive the equality of the HDCs by introducing the moment- and cumulant-generating functions to deal with all the HDCs collectively. Derivations for the zeroth-, first- and second-order parameters are presented in Appendix.

\subsection{Definitions of quantities and assumptions}

We consider component and composite electron swarms in drift equilibrium in gas. The electrons drift under the uniform electric field $-E$ applied in the $x$ direction, satisfying the balance between the energy gain from the electric field and the energy loss by collisions with gas molecules. The composite electron swarm is the sum of the component electron swarms. The quantities of the $k$ th component electron swarm and the composite electron swarm are respectively indicated by subscripts " $k$ " and "total" hereafter. The assumption that each component electron swarm is in drift equilibrium is interpreted as that the transport coefficients are constant and common among the component electron swarms. We denote such common values by symbols with subscript "eq".

From $f_{k}$, we define the $n$ th-order moments $m_{n, k}^{\mathrm{L}}$ in laboratory systems and $m_{n, k}^{\mathrm{C}}$ in centroid systems:

$$
\begin{aligned}
m_{n, k}^{\mathrm{L}}(t) & =\int_{-\infty}^{\infty} x^{n} f_{k}(x, t) \mathrm{d} x, \\
m_{n, k}^{\mathrm{C}}(t) & =\int_{-\infty}^{\infty}\left[x-g_{k}(t)\right]^{n} f_{k}(x, t) \mathrm{d} x, \\
g_{k}(t) & =\frac{m_{1, k}^{\mathrm{L}}(t)}{N_{e, k}(t)},
\end{aligned}
$$

where $g_{k}$ is the centroid of $f_{k}$, and the zeroth-order moments $m_{0, k}^{\mathrm{C}}$ and $m_{0, k}^{\mathrm{L}}$ are both identical to the electron population $N_{\mathrm{e}, k}$.
The effective ionization frequency $R_{k}$, centroid drift velocity $W_{k}$ and $n$ th-order longitudinal diffusion coefficients $D_{n, k}(n \geq 2)$ are defined as ${ }^{1,6)}$

$$
\begin{aligned}
& R_{k}=\frac{\frac{\mathrm{d}}{\mathrm{d} t} N_{\mathrm{e}, k}(t)}{N_{\mathrm{e}, k}(t)}, \\
& W_{k}=\frac{\mathrm{d}}{\mathrm{d} t} \frac{m_{1, k}^{\mathrm{L}}(t)}{N_{e, k}(t)}=\frac{\mathrm{d}}{\mathrm{d} t} g_{k}(t), \\
& D_{n, k}=\frac{1}{n !} \frac{\mathrm{d}}{\mathrm{d} t} \frac{m_{n, k}^{\mathrm{C}}(t)}{N_{e, k}(t)} \quad(\text { for } n=2,3), \\
& D_{n, k}=\frac{1}{n !} \frac{\mathrm{d}}{\mathrm{d} t} \frac{m_{n, k}^{\mathrm{C}}(t)}{N_{e, k}(t)}-\sum_{i=2}^{n-2} \frac{D_{i, k}}{(n-i) !} \frac{m_{n-i, k}^{\mathrm{C}}(t)}{N_{e, k}(t)} \\
& \text { (for } n \geq 4 \text { ). }
\end{aligned}
$$

From the assumption on equilibrium, they satisfy $R_{k}=$ $R_{\mathrm{eq}}, W_{k}=W_{\mathrm{eq}}$ and $D_{n, k}=D_{n, \mathrm{eq}}$ for all $k$ 's, and $N_{\mathrm{e}, k}$ grows exponentially as

$$
N_{e, k}(t)=N_{e, k}(0) \exp \left(R_{k} t\right)=N_{e, k}(0) \exp \left(R_{e q} t\right) .
$$

The quantities of the composite electron swarm are given as

$$
\begin{aligned}
f_{\text {total }}(x, t) & =\sum_{k} f_{k}(x, t) \\
m_{n, \text { total }}^{\mathrm{L}}(t) & =\int_{-\infty}^{\infty} x^{n} f_{\text {total }}(x, t) \mathrm{d} x \\
& =\sum_{k} m_{n, k}^{\mathrm{L}}(t) \\
m_{n, \text { total }}^{\mathrm{C}}(t) & =\int_{-\infty}^{\infty}\left[x-g_{\text {total }}(t)\right]^{n} f_{\text {total }}(x, t) \mathrm{d} x \\
& \neq \sum_{k} m_{n, k}^{\mathrm{C}}(t), \\
g_{\text {total }}(t) & =\frac{m_{1, \text { total }}^{\mathrm{L}}(t)}{N_{e, \text { total }}(t)} \\
& =\frac{\sum_{k} N_{\mathrm{e}, k}(t) g_{k}(t)}{\sum_{k} N_{\mathrm{e}, k}(t)} .
\end{aligned}
$$

With these quantities, $R_{\text {total }}, W_{\text {total }}$ and $D_{n, \text { total }}$ are defined in the same manner as eqs. (6)-(9) for $R_{k}, W_{k}$ and $D_{n, k}$.

\subsection{Relations between moments, cumulants and diffu- sion coefficients}

The moment-generating functions $M_{k}^{\mathrm{L}}$ and $M_{k}^{\mathrm{C}}$ are defined as the series of the moments $m_{n, k}^{\mathrm{L}}$ and $m_{n, k}^{\mathrm{C}}$, respectively, with the parameter $\phi$ as

$$
\begin{aligned}
M_{k}^{\mathrm{L}}(\phi, t) & =\sum_{n=0}^{\infty} \frac{m_{n, k}^{\mathrm{L}}(t)}{n !} \phi^{n}, \\
M_{k}^{\mathrm{C}}(\phi, t) & =\sum_{n=0}^{\infty} \frac{m_{n, k}^{\mathrm{C}}(t)}{n !} \phi^{n} .
\end{aligned}
$$


With the relation $\exp (x \phi)=1+x \phi+\left(x^{2} / 2 !\right) \phi^{2}+$ $\left(x^{3} / 3 !\right) \phi^{3}+\cdots, M_{k}^{\mathrm{L}}$ and $M_{k}^{\mathrm{C}}$ can also be written as

$$
\begin{aligned}
M_{k}^{\mathrm{L}}(\phi, t) & =\int_{-\infty}^{\infty} \exp (x \phi) f_{k}(x, t) \mathrm{d} x, \\
M_{k}^{\mathrm{C}}(\phi, t) & =\int_{-\infty}^{\infty} \exp \left\{\left[x-g_{k}(t)\right] \phi\right\} f_{k}(x, t) \mathrm{d} x \\
& =\exp \left[-g_{k}(t) \phi\right] M_{k}^{\mathrm{L}}(\phi, t) .
\end{aligned}
$$

Moreover, for the composite electron swarm, $M_{\text {total }}^{\mathrm{L}}$ and $M_{\text {total }}^{\mathrm{C}}$ are defined in the same manner as eqs. (15)-(18), using $m_{n, \text { total }}^{\mathrm{L}}, m_{n, \text { total }}^{\mathrm{C}}, f_{\text {total }}$ and $g_{\text {total }}$ instead of $m_{n, k}^{\mathrm{L}}$, $m_{n, k}^{\mathrm{C}}, f_{k}$ and $g_{k}$, respectively.

On the other hand, the cumulant generating function $K$ and cumulant $\kappa_{n}$ are formally defined using the generating function of normalized centered moments as

$$
\begin{aligned}
K_{k}(\phi, t) & =\sum_{n=1}^{\infty} \frac{\kappa_{n, k}(t)}{n !} \phi^{n} \\
& =\ln \frac{M_{k}^{\mathrm{C}}(\phi, t)}{N_{e, k}(t)} \\
K_{\text {total }}(\phi, t) & =\sum_{n=1}^{\infty} \frac{\kappa_{n, \text { total }}(t)}{n !} \phi^{n} \\
& =\ln \frac{M_{\text {total }}^{\mathrm{C}}(\phi, t)}{N_{\text {etotal }}(t)}
\end{aligned}
$$

where $\kappa_{n, k}$ and $\kappa_{n \text {,total }}$ are $n$ th-order cumulants. ${ }^{13-15)}$ The cumulants can be represented with the normalized centered moments $\mu_{n}=m_{n}^{\mathrm{C}} / N_{\mathrm{e}}$ and related to statistical quantities:

$$
\begin{aligned}
& \kappa_{1}=\mu_{1}=0 \quad(=\text { average }), \\
& \kappa_{2}=\mu_{2} \quad\left(=\text { variance }=\sigma^{2}\right), \\
& \kappa_{3}=\mu_{3} \quad\left(c f . \text { skewness }=\kappa_{3} / \sigma^{3}\right), \\
& \kappa_{4}=\mu_{4}-3\left(\mu_{2}\right)^{2} \quad\left(c f . \text { kurtosis }=\kappa_{4} / \sigma^{4}\right), \\
& \kappa_{5}=\mu_{5}-10 \mu_{3} \mu_{2}, \\
& \kappa_{6}=\mu_{6}-15 \mu_{4} \mu_{2}-10\left(\mu_{3}\right)^{2}+30\left(\mu_{2}\right)^{3},
\end{aligned}
$$

where $\sigma$ is the standard deviation. The cumulant is a quantity characterizing the shape of a distribution. When a distribution is a Gaussian, $\kappa_{n}=0$ for $n \geq 3 .{ }^{13,14)}$ Therefore, the higher-order cumulants are regarded as quantities representing the deviation of the distribution from a Gaussian. ${ }^{13)}$ Note here that $\kappa_{n \text {,total may be non- }}$ zero even when $\kappa_{n, k}=0$ for $n \geq 3$ for all $k$ 's. Using $\kappa_{n, k}$ and $\kappa_{n, \text { total }}, D_{n, k}$ and $D_{n, \text { total }}(n \geq 2)$ are given as ${ }^{6)}$

$$
\begin{aligned}
D_{n, k} & =\frac{1}{n !} \frac{\mathrm{d}}{\mathrm{d} t} \kappa_{n, k}(t), \\
D_{n, \text { total }} & =\frac{1}{n !} \frac{\mathrm{d}}{\mathrm{d} t} \kappa_{n, \text { total }}(t) .
\end{aligned}
$$

Figure 2 shows the relations between the moments, cumulants and diffusion coefficients of the component and composite electron swarms. What to be derived is $D_{n, \text { total }}=D_{n, \text { eq }}$ for $n \geq 3$ when $D_{n, k}=D_{n, \text { eq }}$ for all $k$ 's.

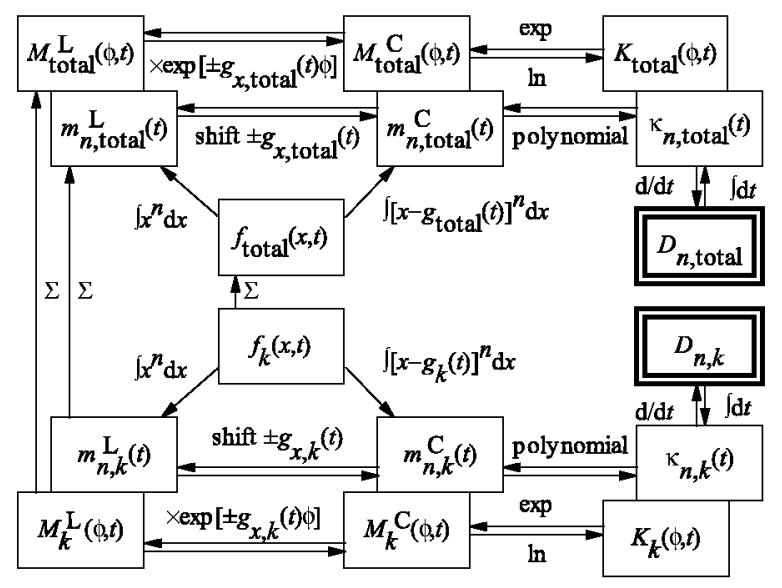

Fig. 2. Relations between quantities concerning the $n$ th-order diffusion coefficients $D_{n}$ : moments in laboratory and centroid systems, $m_{n}^{\mathrm{L}}$ and $m_{n}^{\mathrm{C}}$; cumulants, $\kappa_{n}$; and their generating functions, $M^{\mathrm{L}}, M^{\mathrm{C}}$ and $K$. Subscripts " $k$ " and "total" indicate the $k$ th component and composite electron swarms.

The relation between $D_{n, \text { total }}$ and $D_{n, k}$ is too indirect to realize their equality intuitively. We require several steps of substitutions and transformations of equations, as shown in the next subsection.

\subsection{Derivation of higher-order diffusion coefficient equality}

From eq. (22) and the assumption on equilibrium, we may let

$$
\kappa_{n, k}(t)=n ! D_{n, \mathrm{eq}} t+\kappa_{n, k}(0)
$$

where $\kappa_{n, k}(0)$ is a time-invariant constant. The objective is to show

$$
\kappa_{n, \text { total }}(t)=n ! D_{n, \text { eq }} t+\text { constant. }
$$

We relate $K_{\text {total }}$ and $K_{k}$ through $M_{\text {total }}^{\mathrm{C}}, M_{\text {total }}^{\mathrm{L}}, M_{k}^{\mathrm{L}}$ and $M_{k}^{\mathrm{C}}$ in this sequence. Note that mediation by $M_{\text {total }}^{\mathrm{L}}$ and $M_{k}^{\mathrm{L}}$ is necessary because $M_{\text {total }}^{\mathrm{L}}=\sum_{k} M_{k}^{\mathrm{L}}$ but $M_{\text {total }}^{\mathrm{C}} \neq \sum_{k} M_{k}^{\mathrm{C}} ; m_{n, k}^{\mathrm{C}}$ is defined around $g_{k}$ different for each $k$.

Equation (20) is rewritten as

$$
\begin{aligned}
& M_{\text {total }}^{\mathrm{C}}(\phi, t) \\
& \quad=N_{\text {e,total }}(0) \exp \left(R_{\text {eq }} t\right) \exp \left[K_{\text {total }}(\phi, t)\right] .
\end{aligned}
$$

Links from $M_{\text {total }}^{\mathrm{C}}$ to $K_{k}$ are

$$
\begin{aligned}
M_{\text {total }}^{\mathrm{C}}(\phi, t) & =\exp \left[-g_{\text {total }}(t) \phi\right] M_{\text {total }}^{\mathrm{L}}(\phi, t), \\
M_{\text {total }}^{\mathrm{L}}(\phi, t) & =\sum_{k} M_{k}^{\mathrm{L}}(\phi, t) \\
M_{k}^{\mathrm{L}}(\phi, t) & =\exp \left[g_{k}(t) \phi\right] M_{k}^{\mathrm{C}}(\phi, t), \\
M_{k}^{\mathrm{C}}(\phi, t) & =N_{\mathrm{e}, k}(t) \exp \left[K_{k}(\phi, t)\right] .
\end{aligned}
$$

Thus, we obtain

$$
\begin{aligned}
& M_{\text {total }}^{\mathrm{C}}(\phi, t)=\sum_{k}\left(N_{\mathrm{e}, k}(0) \exp \left(R_{\mathrm{eq}} t\right)\right. \\
& \left.\quad \times \exp \left\{\left[g_{k}(t)-g_{\text {total }}(t)\right] \phi+K_{k}(\phi, t)\right\}\right) .
\end{aligned}
$$


Here, the factor $g_{k}(t)-g_{\text {total }}(t)\left(\equiv d_{k}\right)$ in this equation is time-invariant because the relative velocity between the centroids is zero in equilibrium. Moreover, note that $K_{k}$ has no first-order term because $\kappa_{1, k}=0$. By equating the two expressions of $M_{\text {total }}^{\mathrm{C}}$ in eqs. (26) and (31), we obtain a description of $K_{\text {total }}$ with $\kappa_{n, k}$ :

$$
\begin{aligned}
& \exp \left[K_{\text {total }}(\phi, t)\right]=\sum_{k}\left\{\frac{N_{e, k}(0)}{N_{e, \text { total }}(0)}\right. \\
& \left.\quad \times \exp \left[d_{k} \phi+\frac{\kappa_{2, k}(t)}{2 !} \phi^{2}+\frac{\kappa_{3, k}(t)}{3 !} \phi^{3}+\cdots\right]\right\} .
\end{aligned}
$$

We transform the summation in eq. (32) into a single exponential function to show the dependence of $\kappa_{n, \text { total }}$ on $t$ explicitly. Using eq. (24), the time-variant factor $\exp \left(D_{2, \mathrm{eq}} t \phi^{2}+D_{3, \mathrm{eq}} t \phi^{3}+\cdots\right)$ commonly involved in the exponential functions in the right-hand side of eq. (32) is separated as

$$
\begin{aligned}
& \exp \left[K_{\text {total }}(\phi, t)\right]=\exp \left(D_{2, \text { eq }} t \phi^{2}+D_{3, \text { eq }} t \phi^{3}+\cdots\right) \\
& \quad \times \sum_{k}\left\{\frac{N_{e, k}(0)}{N_{e, \text { total }}(0)}\right. \\
& \left.\quad \times \exp \left[d_{k} \phi+\frac{\kappa_{2, k}(0)}{2 !} \phi^{2}+\frac{\kappa_{3, k}(0)}{3 !} \phi^{3}+\cdots\right]\right\} .
\end{aligned}
$$

The time-invariant factor of eq. (33) is further rewritten by introducing $A_{k}$ and $B$ into the following forms:

$$
\begin{aligned}
\exp \left[K_{\text {total }}(\phi, t)\right] & \\
= & \exp \left(D_{2, \text { eq }} t \phi^{2}+D_{3, \text { eq }} t \phi^{3}+\cdots\right) \\
& \times \sum_{k} \frac{N_{e, k}(0)}{N_{e, \text { total }}(0)} \exp \left[A_{k}(\phi)\right] \\
= & \exp \left(D_{2, \text { eq }} t \phi^{2}+D_{3, \text { eq }} t \phi^{3}+\cdots\right) B(\phi) .
\end{aligned}
$$

$A_{k}$ is a series of $\phi^{n}$ with time-invariant coefficients. Because an expansion of $\exp \left[A_{k}(\phi)\right]$ gives a series of $\left(A_{k}\right)^{i}$ $(i=0,1,2, \cdots)$ as

$$
\begin{array}{r}
\exp \left[A_{k}(\phi)\right]=1+A_{k}(\phi)+\frac{1}{2 !}\left[A_{k}(\phi)\right]^{2} \\
+\frac{1}{3 !}\left[A_{k}(\phi)\right]^{3}+\cdots,
\end{array}
$$

$B$ is also a series of $\phi^{n}$ with time-invariant coefficients. Here, note that the terms of $A_{k}$ are in the first order or higher with respect to $\phi$. Therefore, the constant term free from $\phi$ in eq. (36) is unity, and the constant term in $B$ is also unity since $\sum_{k} N_{e, k}(0) / N_{e, t o t a l}(0)=1$.

At this time, using the formula $\log s=(s-1)-(s-$ $1)^{2} / 2$ ! $+(s-1)^{3} / 3 !-\cdots$ for $0<s<2$, we can rewrite $B$ as

$$
\begin{aligned}
B(\phi)= & \exp [\ln B(\phi)] \\
= & \exp \left\{[B(\phi)-1]-\frac{1}{2 !}[B(\phi)-1]^{2}\right. \\
& \left.\quad+\frac{1}{3 !}[B(\phi)-1]^{3}-\cdots\right\} .
\end{aligned}
$$

Here, we may set the parameter $\phi$ to be as small as necessary to satisfy $0<B(\phi)<2$ by letting $B(\phi) \rightarrow 1$. The exponent in eq. (37) is again a series of $\phi^{n}$, which does not include a constant term free from $\phi$. Introducing the time-invariant coefficients $C_{n}$, we can rewrite $\exp \left[K_{\text {total }}(\phi, t)\right]$ as

$$
\begin{aligned}
& \exp \left[K_{\text {total }}(\phi, t)\right] \\
& =\quad \exp \left(D_{2, \text { eq }} t \phi^{2}+D_{3, \text { eq }} t \phi^{3}+\cdots\right) \\
& \quad \times \exp \left(C_{1} \phi+\frac{C_{2}}{2 !} \phi^{2}+\frac{C_{3}}{3 !} \phi^{3}+\cdots\right) .
\end{aligned}
$$

Therefore,

$$
\begin{aligned}
\exp \left[K_{\text {total }}(\phi, t)\right] & \\
=\exp [ & \kappa_{1, \text { total }}(t) \phi+\frac{\kappa_{2, \text { total }}(t)}{2 !} \phi^{2} \\
& \left.+\frac{\kappa_{3, \text { total }}(t)}{3 !} \phi^{3}+\cdots\right] \\
=\exp \left[C_{1} \phi+\left(D_{2, \text { eq }} t+\frac{C_{2}}{2 !}\right) \phi^{2}\right. & \\
& \left.+\left(D_{3, \text { eq }} t+\frac{C_{3}}{3 !}\right) \phi^{3}+\cdots\right] .
\end{aligned}
$$

By comparing the coefficients of $\phi^{n}$ in eqs. (39) and (40), we obtain eq. (25).

We have shown that the HDCs of the composite electron swarm are equal to those of the component electron swarms. This result has been derived without conditions on the relative position and relative electron population between the component electron swarms. Moreover, the equality of the HDCs holds when the release of the initial electrons is asynchronous among the component electron swarms. For example, the time lag of the initial electron release would appear as the shortened flight distance of the component electron swarms. Therefore, the difference in the release timing is considered as the variation in the spatial arrangement of the component electron swarms.

\section{Numerical Simulations}

\subsection{Simulation methods}

To verify the theoretical result on the equality of the HDCs, we have performed $D_{n}$ calculations up to $n=6$ by a propagator method (PM) and Monte Carlo (MC) simulation. The $\mathrm{PM}^{6,16)}$ is a numerical technique for solving simultaneous moment equations derived from the Boltzmann equation. The $D_{n}$ calculation by the PM was stable. On the other hand, the MC simulates the electron behavior using random numbers. $D_{n}$ calculated by the MC was severely influenced by the statistical fluctuation, as discussed afterward. Equilibrium $D_{n}$ values were recognizable up to $n=4$ in the MC with electron samplings of $10^{7}-10^{8}$.

\subsection{Simulation conditions}

$\mathrm{CF}_{4}$ has been chosen as a test gas. $\mathrm{CF}_{4}$ has both ionization and attachment processes, and its vibrational excitation cross section lying in a low-energy region is expected to make the spatial electron distribution shape of 


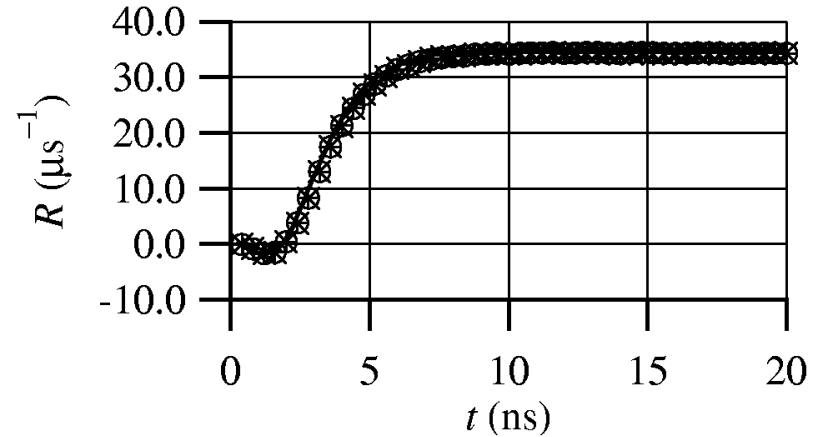

Fig. 3. Effective ionization frequencies of component and composite electron swarms:,$+ R_{1}(\mathrm{MC}) ; \times, R_{2}(\mathrm{MC}) ; \circ, R_{\text {total }}$ (MC); - $R_{1}, R_{2}$ and $R_{\text {total }}$ (PM, overlapping).

the electron swarms complicated. The electron collision cross section set for $\mathrm{CF}_{4}$ has been taken from ref. 17 . The gas molecule number density $N$ has been set at $3.54 \times 10^{16} \mathrm{~cm}^{-3}$, assuming the gas pressure $p=133 \mathrm{~Pa}$ ( 1 Torr) at $0^{\circ} \mathrm{C}$, and the applied electric field was $E=$ $100.0 \mathrm{~V} \mathrm{~cm}^{-1}$. These values correspond to the reduced electric field $E / N$ of $283 \mathrm{Td}\left(1 \mathrm{Td}=10^{-17} \mathrm{~V} \mathrm{~cm}^{2}\right)$.

The initial electrons have been released from two point electron sources, $\mathrm{P}_{1}$ at $(x, y, z)=(0,0,0)$ and $\mathrm{P}_{2}$ at $(0.5 \mathrm{~cm}, 1.0 \mathrm{~cm}, 1.5 \mathrm{~cm})$, at $t=0$ with an uneven ratio of $60: 40$. This setting supposes a composite electron swarm consisting of two component electron swarms. The quantities concerning the $x$ direction are the objects of the present interest. The energy distribution of the initial electrons was assumed to be a Maxwellian of the mean electron energy of $1.0 \mathrm{eV}$. The electron swarms were traced until $t=40 \mathrm{~ns}$, which is sufficiently long for the transport parameters to reach their equilibrium values.

\section{Results and Discussion}

\subsection{Transport parameters}

Figures 3-7 show the relaxation processes of $R, W$, $D_{2}, D_{3}$ and $D_{4}$ of the component and composite electron swarms. It seems that their values have reached equilibrium by $t=20 \mathrm{~ns}$. The transport parameters of the composite electron swarm agree well with those of the component electron swarms. The statistical fluctuations in $D_{5}$ and $D_{6}$ sampled by the MC were too large to recognize their equilibrium values. The equality of $D_{5}$ and $D_{6}$ was verified by the result of the PM (Figs. 8 and 9).

Figures 10 and 11 show the temporal variations in $\kappa_{2}$ and $\kappa_{3}$ of the component and composite electron swarms. The difference between the initial $\kappa_{n, k}$ and $\kappa_{n \text {,total values }}$ represents the difference between the shapes of $f_{k}$ and $f_{\text {total }}$. However, their time derivatives are common and constant in equilibrium. This result verifies eqs. (24) and (25).

The tendency observed in the MC result is that the statistical fluctuation is larger in the composite electron swarm than in the component electron swarms (see $D_{4}$ in Fig. 7), although the composite electron swarm contains more electrons than each component electron swarm. This is considered to be due to the fact that the distance

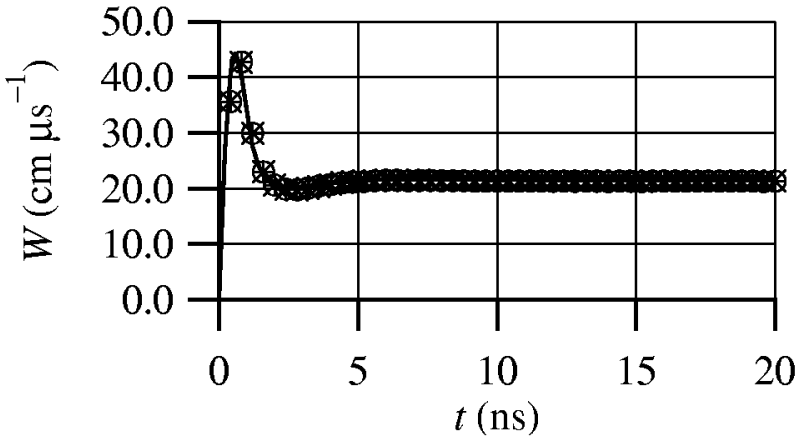

Fig. 4. Drift velocities of component and composite electron swarms:,$+ W_{1}(\mathrm{MC}) ; \times, W_{2}(\mathrm{MC}) ; \circ, W_{\text {total }}(\mathrm{MC}) ;-, W_{1}$, $W_{2}$ and $W_{\text {total }}$ (PM, overlapping).

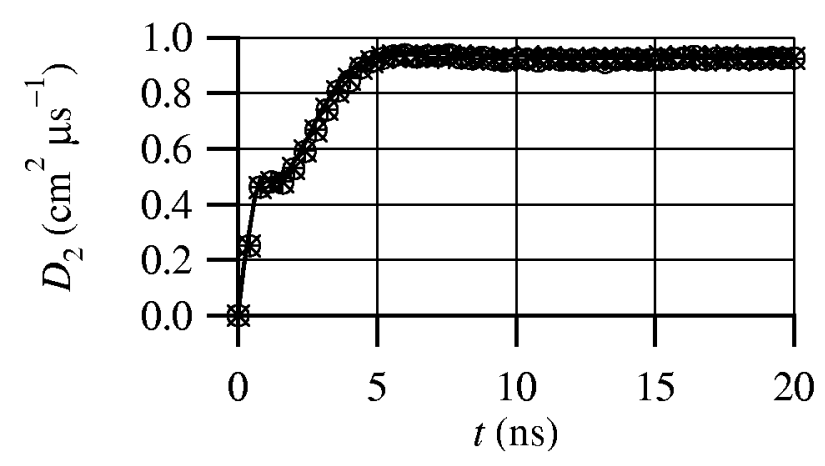

Fig. 5. Second-order diffusion coefficients of component and composite electron swarms:,$+ D_{2,1}(\mathrm{MC}) ; \times, D_{2,2}(\mathrm{MC}) ; \circ, D_{2, \text { total }}$

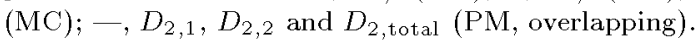

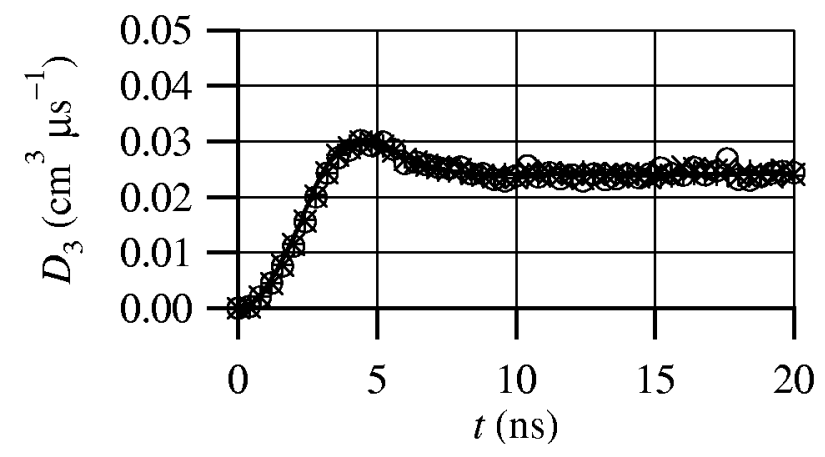

Fig. 6. Third-order diffusion coefficients of component and composite electron swarms:,$+ D_{3,1}(\mathrm{MC}) ; \times, D_{3,2}(\mathrm{MC}) ; 0, D_{3, \text { total }}$

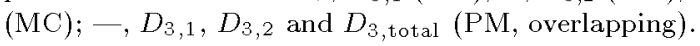

factor $\left[x-g_{\text {total }}(t)\right]^{n}$ in eq. (13) for the composite electron swarm is larger than $\left[x-g_{k}(t)\right]^{n}$ in eq. (4) for each component electron swarm. $D_{n}$ becomes more sensitive to this distance factor as $n$ increases. The fluctuations in $D_{4, \text { total }}, D_{4,1}$ and $D_{4,2}$ increase with $t$ probably for the same reason. The distance from the centroid also increases for electron diffusion. Although the exponential increase in $N_{\mathrm{e}}$ due to ionization often stabilizes the MC sampling result, the instability due to the statistical fluctuation appears strongly in the calculation of the HDCs. 


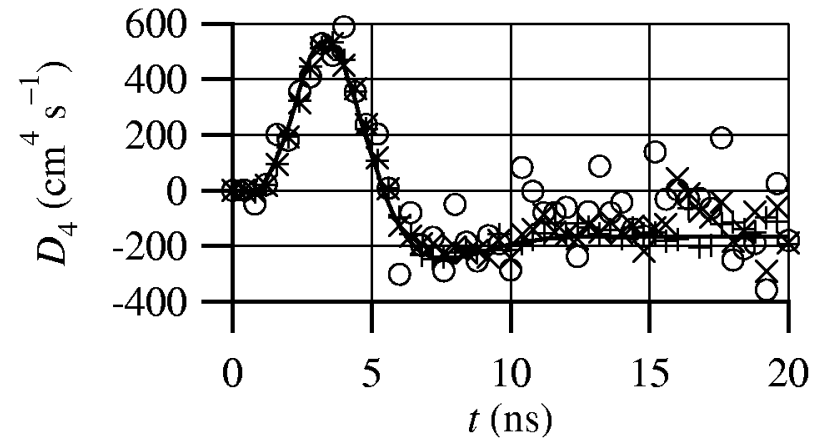

Fig. 7. Fourth-order diffusion coefficients of component and composite electron swarms:,$+ D_{4,1}(\mathrm{MC}) ; \times, D_{4,2}(\mathrm{MC}) ; \circ, D_{4, \text { total }}$

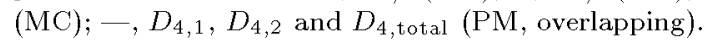

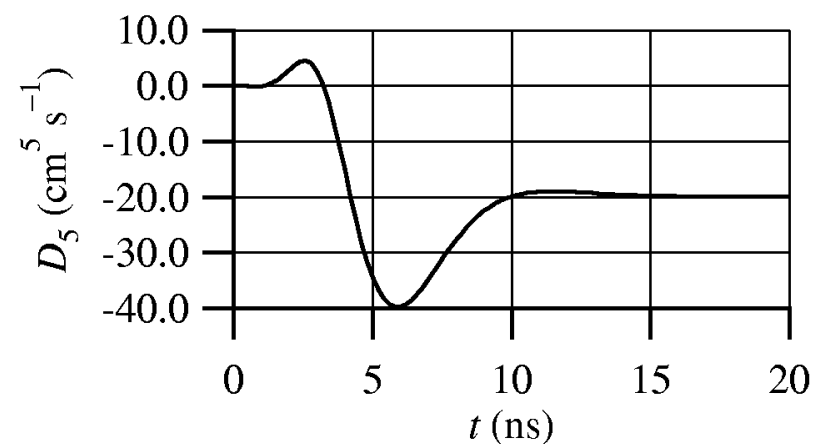

Fig. 8. Fifth-order diffusion coefficients of component and composite electron swarms calculated by PM: $D_{5,1}, D_{5,2}$ and $D_{5, \text { total }}$ (overlapping).

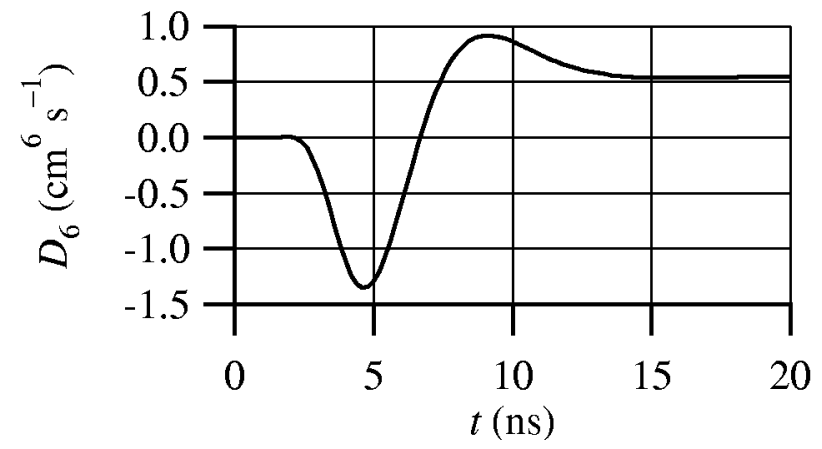

Fig. 9. Sixth-order diffusion coefficients of component and composite electron swarms calculated by PM: $D_{6,1}, D_{6,2}$ and $D_{6, \text { total }}$ (overlapping).

\subsection{Spatial electron distribution}

Figure 12 shows the development of $f_{\text {total }}$ composed by $f_{1}$ and $f_{2}$. The shape of $f_{\text {total }}$ is still far from a Gaussian at $t=20 \mathrm{~ns}$, while the transport parameters of the component and composite electron swarms have reached their equilibrium values by this moment. This supports one of the theoretical results that the equality of the transport parameters is independent of the spatial arrangement of the component electron swarms.

It is generally thought that the shape of the spatial

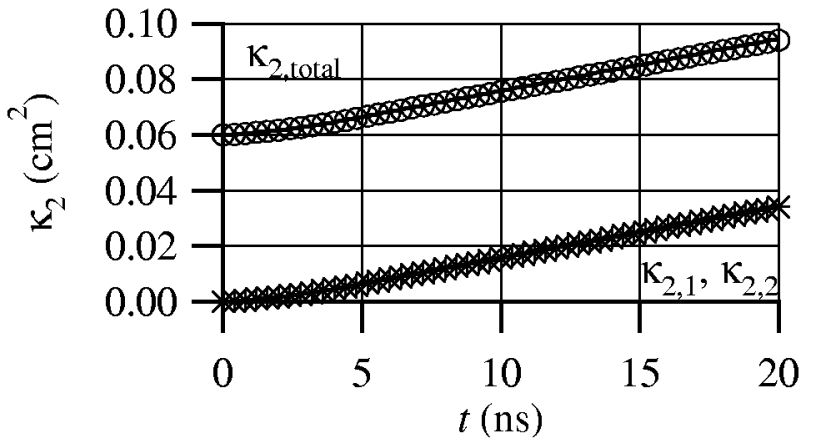

Fig. 10. Second-order cumulants of component and composite electron swarms:,$+ \kappa_{2,1}(\mathrm{MC}) ; \times, \kappa_{2,2}(\mathrm{MC}) ; \circ, \kappa_{2, \text { total }}(\mathrm{MC})$; - $\kappa_{2,1}, \kappa_{2,2}$ and $\kappa_{2, \text { total }}(\mathrm{PM})$.

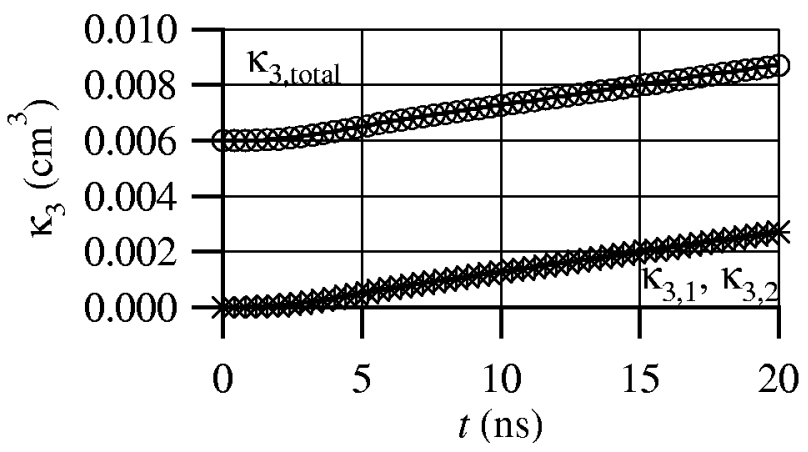

Fig. 11. Third-order cumulants of component and composite electron swarms:,$+ \kappa_{3,1}(\mathrm{MC}) ; \times, \kappa_{3,2}$ (MC); o, $\kappa_{3, \text { total }}$ (MC); -, $\kappa_{3,1}, \kappa_{3,2}$ and $\kappa_{3, \text { total }}(\mathrm{PM})$.

electron distribution of an electron swarm tends to be a Gaussian. Sugawara et al. ${ }^{5)}$ theoretically showed this tendency for an isolated electron swarm developing from a point electron source. This tendency is also observed in $f_{\text {total }}$ of the composite electron swarm in Fig. 12. As the electrons diffuse, the distance between $\mathrm{P}_{1}$ and $\mathrm{P}_{2}$ would eventually become negligible relative to a spatial scale (e.g., the standard deviation $\sigma=\sqrt{\kappa_{2, \text { total }}}$ ) of $f_{\text {total }}$. This is a basis with which we can approximate the electron source as a point in swarm experiments. Here, this tendency does not conflict with the infinite increase of $\mid \kappa_{n}$,total $\mid$ with $t$ represented by eq. (25), although $\kappa_{n \text {,total }}$ is a quantity characterizing the deviation of $f_{\text {total }}$ from a Gaussian. Sugawara et al. ${ }^{6)}$ showed that while the absolute amount of the deviation increases, its dimensionless value normalized by $\sigma$ tends to be zero.

\subsection{Higher-order transverse diffusion coefficients}

The derivation of the equality of $D_{n}$ presented in $\S 2$ can also be applied to the transverse diffusion coefficient $D_{\mathrm{T} n}(n \geq 2)$. $D_{\mathrm{T} n}$ quantifies the electron diffusion in the $y$ direction lateral to the applied electric field and is defined in the same manner as that for $D_{n}$; the definition of $D_{n}$ becomes that of $D_{\mathrm{T} n}$ by replacing $x$ with $y$. Figure 13 shows the schematic of $f_{\text {total }}(y)$ under the present simulation conditions.

Date t $a l .{ }^{18)}$ derived the characteristic feature of $D_{\mathrm{T} n}$ 


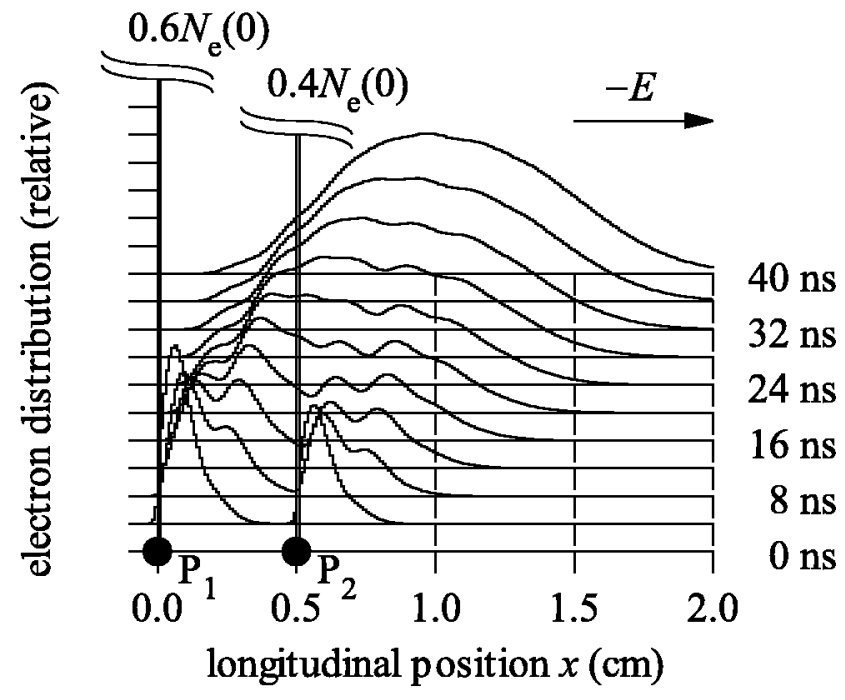

Fig. 12. Spatiotemporal development of electron swarm in $\mathrm{CF}_{4}$ calculated by $\mathrm{MC}$ at $E / N=283 \mathrm{Td}$. Sixty percent of the initial electrons has been released from electron source $\mathrm{P}_{1}$ at $x=0 \mathrm{~cm}$, and forty percent from electron source $\mathrm{P}_{2}$ at $x=0.5 \mathrm{~cm}$.

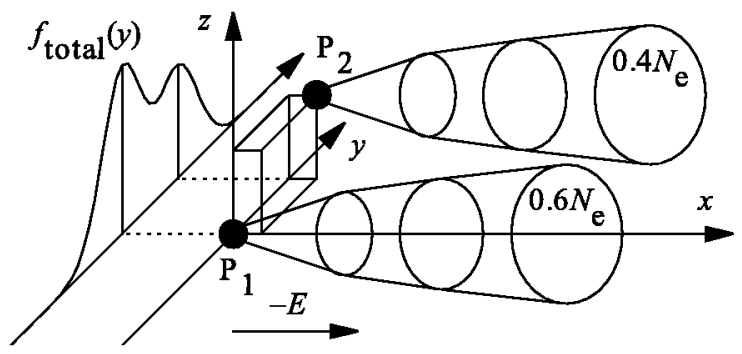

Fig. 13. Schematic of asymmetrical transverse electron distribution.

by Fourier-transformed Boltzmann equation analysis:

$$
D_{\text {T } n, \text { total }}=0 \quad(\text { for } n=3,5,7, \cdots) .
$$

This feature can also be derived from the equality of $D_{\mathrm{T} n}$ between the component and composite electron swarms. Equation (41) is evident when $f_{\text {total }}(y)$ itself is symmetrical in $y=g_{\mathrm{T}, \text { total }}$, because $D_{\mathrm{T} n}$ 's $(n=3,5,7, \cdots)$ are given by integrations for the odd functions of $y .{ }^{6,19}$ ) Moreover, $f_{\text {total }}(y)$ may be asymmetrical as long as its components $f_{k}(y)$ are all individually symmetrical, because $D_{\mathrm{T} n, \text { total }}=0$ for odd $n$ when $D_{\mathrm{T} n, k}=0$ for all $k$ 's (the equality of $D_{\mathrm{T} n}$ ). Figure 14 shows $D_{\mathrm{T} 3, k}$ and $D_{\mathrm{T} 3 \text {,total }}$ calculated by the $\mathrm{MC}$ for the same component and composite electron swarms as have been examined. While each component electron swarm is symmetrical, the composite electron swarm is asymmetrical. Comparing with $D_{3}$ plotted together, we can observe that $D_{\mathrm{T} 3, k}=D_{\mathrm{T} 3, \text { total }}=0$.

Furthermore, it is considered that the symmetry of $f_{k}(y)$ is always established in equilibrium even though the initial electrons are given with asymmetrical spatial and velocity distributions. Let us assume that the $k$ th initial electron generates the $k$ th component electron swarm and that electron scattering by gas molecules

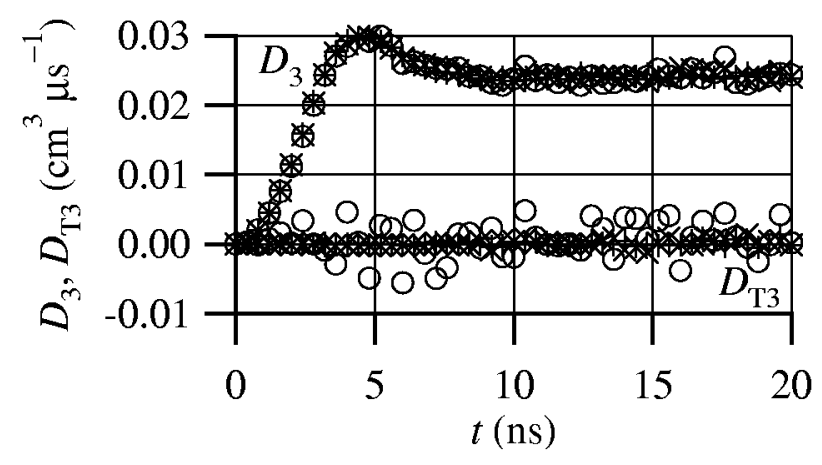

Fig. 14. Third-order longitudinal and transverse diffusion coefficients of component and composite electron swarms calculated by $\mathrm{MC}:+, D_{3,1}$ and $D_{\mathrm{T} 3,1} ; \times, D_{3,2}$ and $D_{\mathrm{T} 3,2} ;$ and $\circ, D_{3, \text { total }}$ and $D_{\mathrm{T} 3, \text { total }}$.

is isotropic. The expectation of $f_{k}(y)$ becomes symmetrical after the first collision of the $k$ th initial electron, because the asymmetry in the velocity vector of the initial electron vanishes from the expectation of $f_{k}(y)$ at the scattering. Consequently, eq. (41) always holds even in asymmetrical electron swarms. Actually, eq. (41) was derived in ref. 18 with no assumption on the shape of $f_{\text {total }}(y)$.

\section{Conclusions}

A theorem on the higher-order diffusion coefficients (HDCs) has been derived; the $n$ th-order diffusion coefficient $D_{n}(n \geq 2)$ of a composite electron swarm consisting of component electron swarms are equal to that of the component electron swarms when they are individually in drift equilibrium. This theorem represents the equality of the HDCs between an electron swarm developing from multiple electron sources and another originating from a single electron source. Although the HDCs are derived from the higher-order cumulants characterizing the shape of the spatial electron distribution of the composite electron swarm, the equality of the HDCs holds irrespective of the spatial arrangement and electron population of the component electron swarms.

The HDCs of a composite electron swarm consisting of two component electron swarms have been calculated by a propagator method and a Monte Carlo simulation. It has been numerically demonstrated that $D_{n}$ 's up to $n=6$ are equal between the component and composite electron swarms.

Furthermore, the present theorem is applicable not only to the longitudinal diffusion coefficients $D_{n}$ but also to the transverse diffusion coefficients $D_{\mathrm{T} n}$. The fact that $D_{\mathrm{T} n}=0$ for $n=3,5,7, \cdots$ has been explained from the equality of the HDCs.

\section{Acknowledgement}

This work was partly supported by a Grant-in-Aid from the Ministry of Education, Culture, Sports, Science and Technology. 


\section{Appendix: Equality of Lower-Order Transport} Parameters

The equalities of the zeroth-order $\left(R_{\text {total }}=R_{\text {eq }}\right)$ and the first-order $\left(W_{\text {total }}=W_{\text {eq }}\right)$ parameters are almost evident. The former is based on the exponential growth of $N_{\text {e,total }}$ derived from eq. (10):

$$
\begin{aligned}
N_{\mathrm{e}, \text { total }}(t) & =\sum_{k} N_{\mathrm{e}, k}(0) \exp \left(R_{\mathrm{eq}} t\right) \\
& =N_{\mathrm{e}, \text { total }}(0) \exp \left(R_{\mathrm{eq}} t\right), \\
R_{\text {total }} & =\frac{\frac{\mathrm{d}}{\mathrm{d} t} N_{\mathrm{e}, \text { total }}(t)}{N_{\mathrm{e}, \text { total }}(t)}=R_{\mathrm{eq}} .
\end{aligned}
$$

The latter is concluded from $W_{k}=W_{\text {eq }}$ for all $k$ 's; i.e., the relative velocity between $g_{k}$ 's of any two component electron swarms is zero. With eqs. (14) and (A.1), $W_{\text {total }}$ is equated to $W_{\mathrm{eq}}$ as

$$
\begin{aligned}
g_{\text {total }}(t) & =\frac{\sum_{k} N_{\mathrm{e}, k}(0) \exp \left(R_{\mathrm{eq}} t\right) g_{k}(t)}{N_{e, \text { total }}(0) \exp \left(R_{\mathrm{eq}} t\right)} \\
& =\frac{\sum_{k} N_{\mathrm{e}, k}(0) g_{k}(t)}{N_{\mathrm{e}, \text { total }}(0)} \\
W_{\text {total }} & =\frac{\sum_{k} N_{\mathrm{e}, k}(0) \frac{\mathrm{d}}{\mathrm{d} t} g_{k}(t)}{N_{\mathrm{e}, \text { total }}(0)} \\
& =\frac{\sum_{k} N_{\mathrm{e}, k}(0) W_{\mathrm{eq}}}{N_{\mathrm{e}, \text { total }}(0)}=W_{\mathrm{eq}} .
\end{aligned}
$$

The equality of the second-order parameter $\left(D_{2, \text { total }}=\right.$ $D_{2, \text { eq }}$ ) is not immediately evident, because $m_{2, \text { total }}^{\mathrm{C}}$ for deriving $D_{2}$,total involves the distance from the centroid as a nonlinear weight:

$$
\begin{aligned}
D_{2, k} & =\frac{1}{2} \frac{\mathrm{d}}{\mathrm{d} t} \frac{m_{2, k}^{\mathrm{C}}(t)}{N_{e, k}(t)} \\
& =\frac{1}{2} \frac{\mathrm{d}}{\mathrm{d} t} \frac{\int_{-\infty}^{\infty}\left[x-g_{k}(t)\right]^{2} f_{k}(x, t) \mathrm{d} x}{N_{\mathrm{e}, k}(t)} \\
& =D_{2, \mathrm{eq}}, \\
D_{2, \text { total }} & =\frac{1}{2} \frac{\mathrm{d}}{\mathrm{d} t} \frac{m_{2, \text { total }}^{\mathrm{C}}(t)}{N_{\mathrm{e}, \text { total }}(t)} \\
& =\frac{1}{2} \frac{\mathrm{d}}{\mathrm{d} t} \frac{\int_{-\infty}^{\infty}\left[x-g_{\text {total }}(t)\right]^{2} f_{\text {total }}(x, t) \mathrm{d} x}{N_{\mathrm{e}, \text { total }}(t)} .
\end{aligned}
$$

The shape of $f_{\text {total }}$ is dependent on the arrangement of $f_{k}$, and $f_{k}$ may be placed anywhere relative to the other component electron swarms. However, $D_{2, \text { total }}=D_{2, \text { eq }}$ is derived independently of this arrangement.

Expanding the factor $\left[x-g_{k}(t)\right]^{2}$ in eq. (A.5) and integrating its result, we obtain

$$
\begin{aligned}
& D_{2, k}=\frac{1}{2} \frac{\mathrm{d}}{\mathrm{d} t}\left\{\frac{m_{2, k}^{\mathrm{L}}(t)}{N_{e, k}(t)}-\left[g_{k}(t)\right]^{2}\right\} \\
& =\frac{1}{2} \frac{\frac{\mathrm{d}}{\mathrm{d} t} m_{2, k}^{\mathrm{L}}(t)-R_{\mathrm{eq}} m_{2, k}^{\mathrm{L}}(t)}{N_{\mathrm{e}, k}(t)}-W_{\mathrm{eq}} g_{k}(t) .
\end{aligned}
$$

In the same manner, from eq. (A.6), $D_{2, \text { total }}$ is rewritten as

$$
\begin{aligned}
D_{2, \text { total }}= & \frac{1}{2} \frac{\frac{\mathrm{d}}{\mathrm{d} t} m_{2, \text { total }}^{\mathrm{L}}(t)-R_{\mathrm{eq}} m_{2, \text { total }}^{\mathrm{L}}(t)}{N_{\mathrm{e}, \text { total }}(t)} \\
& -W_{\text {eq }} g_{\text {total }}(t) .
\end{aligned}
$$

By decomposing $m_{2 \text {,total }}^{\mathrm{L}}$ and $g_{\text {total }}$ into $m_{2, k}^{\mathrm{L}}$ and $g_{k}$, this equation becomes

$$
\begin{aligned}
D_{2, \text { total }} & \frac{1}{2} \frac{\frac{\mathrm{d}}{\mathrm{d} t} \sum_{k} m_{2, k}^{\mathrm{L}}(t)-R_{\mathrm{eq}} \sum_{k} m_{2, k}^{\mathrm{L}}(t)}{N_{e, \text { total }}(t)} \\
& -W_{\mathrm{eq}} \frac{\sum_{k} N_{\mathrm{e}, k}(t) g_{k}(t)}{N_{\mathrm{e}, \mathrm{total}}(t)} \\
= & \sum_{k}\left\{\frac{N_{\mathrm{e}, k}(t)}{N_{\mathrm{e}, \mathrm{total}}(t)}\right. \\
& \left.\times\left[\frac{1}{2} \frac{\frac{\mathrm{d}}{\mathrm{d} t} m_{2, k}^{\mathrm{L}}(t)-R_{\mathrm{eq}} m_{2, k}^{\mathrm{L}}(t)}{N_{\mathrm{e}, k}(t)}-W_{\mathrm{eq}} g_{k}(t)\right]\right\} .
\end{aligned}
$$

Because the terms in brackets in eq. (A.9) are identical to the description of $D_{2, k}$ in eq. (A.7), we obtain

$$
D_{2, \text { total }}=\frac{\sum_{k} N_{e, k}(t) D_{2, \mathrm{eq}}}{N_{\mathrm{e}, \text { total }}(t)}=D_{2, \mathrm{eq}} .
$$

1) H. Tagashira, Y. Sakai and S. Sakamoto: J. Phys. D 10 (1977) 1051.

2) K. Kumar, H. R. Skullerud and R. E. Robson: Aust. J. Phys, 33 (1980) 343.

3) H. A. Blevin and J. Fletcher: Aust. J. Phys. 37 (1984) 593.

4) J. H. Whealton and E. A. Mason: Ann. Phys. (N. Y.) 84 (1974) 8.

5) J. H. Whealton: J. Phys. B 7 (1974) 1602

6) H. Sugawara, Y. Sakai, H. Tagashira and K. Kitamori: J. Phys. D 31 (1998) 319.

7) J. de Urquijo, C. A. Arriaga, C. Cisneros and I. Alvarez: J. Phys. D 32 (1999) 41.

8) J. de Urquijo: Plasma Source Sci. Technol. 11 (2002) A86.

9) J. de Urquijo, J. L. Hernández-Ávila, E. Basurto and F. Ramírez: J. Phys. D 36 (2003) 1489.

10) D. M. Xiao, L. L. Zhu and Y. Z. Chen: J. Phys. D 32 (1999) L18.

11) D. M. Xiao, L. L. Zhu and X. G. Li: J. Phys. D 33 (2000) L145.

12) Y. Nakamura: J. Phys. D 20 (1987) 933.

13) Suugaku Jiten (Encyclopedia of Mathematics), ed. Mathematical Society of Japan (Iwanami, Tokyo, 1985) p. 853 [in Japanese].

14) W. C. Rinaman, C. Heil, M. T. Strauss, M. Mascagni and M. Sousa: in Standard Mathematical Tables and Formulae: Probability and Statistics, ed. D. Zwillinger (CRC Press, Boca Raton, 1996) 30th ed., Chap. 7, p. 574.

$15)$ E. W. Weisstein: $C R C$ Concise Encyclopedia of Mathematics, ed. E. W. Weisstein (CRC Press, Boca Raton, 1999) p. 370.

16) H. Sugawara and Y. Sakai: J. Phys. D 32 (1999) 1671.

17) M.-C.Bordage, P. Ségur, L. G. Christophorou and J.K. Olthoff: J. Appl. Phys. 86 (1999) 3558.

18) H. Date, K. Kondo and H. Tagashira: J. Phys. D 26 (1993) 1211.

19) K. Kitamori, H. Tagashira and Y. Sakai: J. Phys. D 13 (1980) 535. 